\title{
Local to Extended Transitions of Resonant Defect Modes
}

\author{
Joseph Lydon, ${ }^{1}$ Marc Serra-Garcia, ${ }^{2}$ and Chiara Daraio ${ }^{1,2, *}$ \\ ${ }^{1}$ Engineering and Applied Science, California Institute of Technology, Pasadena, California 91125, USA \\ ${ }^{2}$ Department of Mechanical and Process Engineering, Swiss Federal Institute of Technology (ETH), Zürich 8092, Switzerland
}

(Received 6 June 2014; published 29 October 2014)

\begin{abstract}
We study the localized modes created by introducing a resonant defect in a mechanical lattice. We find that modes introduced by resonant defects have profiles that can be tuned from being extremely localized to totally delocalized by an external force. This is in direct contrast with modes introduced by traditional mass or stiffness defects, in which the modes' profiles stay constant. We present an analytical model for resonant defects in one-dimensional nonlinear lattices, computationally demonstrate the equivalent effect in a twodimensional lattice, and experimentally observe the mode profiles in a granular crystal. While our study is concerned with nonlinear mechanical lattices, the generality of our model suggests that the same effect should be present in other types of periodic lattices.
\end{abstract}

DOI: 10.1103/PhysRevLett.113.185503

PACS numbers: 63.20.Pw, 43.40.+s, 63.20.Ry

Introduction.-The ability to actively control a material's properties through external stimuli is rare and is a goal of material design $[1,2]$. Defects have an enormous effect on material properties: electrical conductivity in semiconductors [3], thermal conductivity [4], and mechanical strength $[5,6]$ are just a few examples. This is in part because defects in periodic lattices allow for local modes of vibration that interact with propagating waves and affect material properties [7-10].

The periodicity of crystal lattices may result in frequency bands and band gaps [11]. Modes that are in the frequency band extend the entire length of the crystal. In contrast, a mode with frequency in the band gap is not extended but spatially localized. A defect breaks the symmetry of the lattice and can introduce such a localized mode. The mode's spatial extension depends on where its frequency lies in the band gap. As the mode's frequency gets closer to a band, the mode profile becomes more delocalized. In traditional mass or stiffness defects, external effects that shift the band frequencies also introduce a proportional shift in the defect mode frequency, rendering the profile of the defect mode insensitive to external influences. This means that for most defects, the localization is determined by the defect to lattice mass ratio [12]. We show that for a resonant defect the prescribed relationship between mode frequency and band frequency does not exist, and hence the mode profile can be tuned by an external force. This is due to an additional degree of freedom provided by the displacement of the secondary mass [13]. Control over the localization is interesting to applications of defect modes [14-17], since many properties, for example, the wave speed in coupled resonant optical waveguides $[16,18]$ and the coupling of phonon-photon interactions [14], depend on the spatial overlap of modes.

The ability to tune frequency bands through external stimuli has its origin in nonlinear interactions. In a granular chain the nonlinearity comes from the Hertzian contact law [19]. However, analogous tuning is possible in other lattices. For example, by applying strain for electronic, phononic, and photonic bands in silicon [20] and graphene [21-23], magnetic fields for electronic bands in carbon nanotubes [24], or also, electric fields [25] and heating [26] for photonic bands in liquid crystals. By placing a resonant defect in a lattice with this type of band tunablility, the defect's local mode can be controlled through an external stimulus. We demonstrate this in a nonlinear mechanical lattice. A compression changes the coupling stiffness of the lattice and, therefore, also the frequency band $[19,27]$. A resonant defect breaks the constant relationship between the defect mode's frequency $f_{d}$, and the band's cutoff frequency $f_{c}$. We can therefore adjust the localization of the defect mode, allowing the local mode profile to resemble mode profiles resulting from defects of differing masses. Figure 1 schematically illustrates this concept.

Experimental system.-The nonlinear mechanical system we use to study this phenomenon is a granular crystal, a lattice of spherical particles coupled through nonlinear Hertzian contacts [28]. Granular crystals have previously been a test bed to study physical phenomena including acoustic rectification [29], discrete breathers [30], acoustic focusing [31] and tunable filtering [27]. Their macroscopic size allows visualization of a lattice's defect dynamics [12], and, therefore, they provide an ideal test lattice for our study.

We introduce a resonant defect in the granular chain by attaching an external ring structure to one of the particles [32]. This structure has a mechanical resonance measured experimentally at $6.2 \mathrm{kHz}$. We embed this resonant defect in the center of a 31-particle chain, with 15 stainless steel spheres (type 316, $9.525 \mathrm{~mm}$ radius) placed on both sides of the resonant defect. This is a one-dimensional finite crystal with a defect in the center. Using 2 polycarbonate 


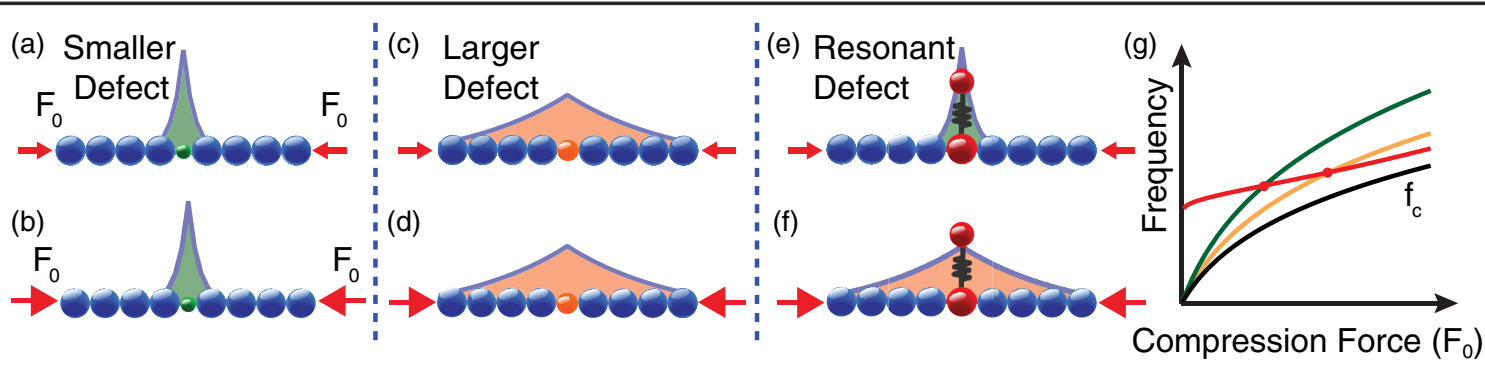

FIG. 1 (color online). A schematic illustrating defect induced local modes and resonant defect tunability. (a),(c), and (e) represent the lattice subject to lower compression, while (b),(d), and (f) represent a higher compression. (a),(b) A mode due to a small defect (green) that is highly localized and does not change its profile between low (a) and high compression (b). (c), (d) Show that the mode due to a larger (red) defect is less localized and still does not depend on compression. (e) and (f) A mode created by a resonant defect (red) in which the localization can be tuned to from highly localized to completely delocalized. $(\mathrm{g})$ Illustrates the origin of this tunability. The small (green) and large (orange) defect frequencies maintain a constant proportion with the band cutoff frequency, resulting in an unchanged localization. In the case of the resonant defect (red) frequency, this constant proportionality is not prescribed. Thus, compression changes the spatial profile of a resonant defect mode.

rods, the particles are aligned between a $90 \mu \mathrm{m}$ compression actuator (Physik Instrumente P-841.60) and static force sensor (Omega LCMFD-50N). A laser vibrometer (Polytec OFV-534) sequentially measures velocities of half the chain and the defect particle. Embedded force sensors are placed next to the defect and at the end of the chain. All dynamic measurements are taken at steady state using a lock-in amplifier (Zurich Instruments ZIHFLI). We drive the defect's resonating mass and measure the forces and velocities of different particles in the lattice.

Theoretical model.-We model our system as masses coupled by springs with a single defect site. All particles other than the defect are spherical with $R=9.525 \mathrm{~mm}$ and a mass $m_{s}=28.8 \mathrm{~g}$. The equations of motion are

$$
\begin{aligned}
& m_{s} \ddot{u}_{i}=k_{c}\left(u_{i+1}+u_{i-1}-2 u_{i}\right) ; \quad i \neq 0, \\
& m_{0} \ddot{u}_{0}=k_{c}\left(u_{1}+u_{-1}-2 u_{0}\right)+k_{R}\left(u_{r}-u_{0}\right), \\
& m_{r} \ddot{u}_{r}=k_{r}\left(u_{0}-u_{r}\right),
\end{aligned}
$$

where $u_{i}$ is the displacement of the $i$ th sphere around its equilibrium position. The equations represent a onedimensional lattice with a local resonance at the defect site. The defect's dynamics are contained in the second two equations and the rest of the lattice in the first. The defect has a mass located in the chain array which is $m_{0}=$ $47.8 \mathrm{~g}$ and has a displacement from equilibrium $u_{0}$. The defect's resonant mass $m_{r}=0.18 \mathrm{~g}$ is coupled with a stiffness $k_{r}=2.64 \times 10^{5} \mathrm{~N} / \mathrm{m} \quad$ (see Supplemental Material [33]) to this particle and has a displacement $u_{r}$. This displacement is the additional degree of freedom for the resonator. We define the characteristic frequency of the resonator $f_{r}=\sqrt{k_{r} / m_{r}}$, which is independent of the lattice, and we stress that this is not equal to the defect mode's frequency. The stiffness coupling all other particles within the chain is found by linearizing the Hertzian contact law $k_{c}=\frac{3}{2} A^{2 / 3} F_{0}^{1 / 3}\left[N^{2 / 3} / \mu m\right]$, where $F_{0}$ is the compression, $A=\frac{4}{3} \sqrt{(R / 2)}\left(E / 2\left(1-\nu^{2}\right)\right), E=193 \mathrm{GPa}$, and $\nu=0.3$ [34]. This is where the nonlinear nature of our lattice is important. It allows a change in the coupling stiffness between adjacent particles through compression, while the resonant coupling stiffness $k_{r}$ stays constant. Finally, the boundary conditions are modeled as fixed walls with $u_{-16}=u_{16}=0$.

The dynamics we are interested in are linear and, therefore, we solve the eigenvalue problem that results from assuming oscillatory solutions. In the Supplemental Material [33] we show our analytical derivation, which is used to fit to experiments and demonstrate that boundary conditions do not become important until just before the mode transitions from local to extended dynamics.

Tuning mode profiles. - We study the dynamics of a local mode and observe how it transitions from highly localized to completely delocalized as a result of an external compression. Figure 2 shows the steady state force measured in the particle next to the resonant defect [Fig. 2(a)] and at the end of the chain [Fig. 2(b)]. This dynamic force results from a harmonic excitation at the defect's resonating mass. The measured force is a function of the excitation frequency and static compression. On top of the experimental results, the theoretical expression for the band edge in a granular crystal is plotted as a white dotted line, $f_{c}=4.9 F_{0}^{1 / 6}[\mathrm{kHz}]$. Below this line, we see the individual modes of the band rise up in frequency as the compression increases. Since the force sensor in Fig. 2(a) is next to the defect, this measures both local and extended dynamic forces. Figure 2(a) shows the presence of the defect resonance mode (varying slightly around $6.2 \mathrm{kHz}$ ) at all compressions. This defect mode frequency increases only a few $\mathrm{Hz}$ as a result of increasing compression. This is due to the small mass participation $m_{r}$ of the resonating structure. Figure 2(b) shows the force in the sensor at the 
(a)
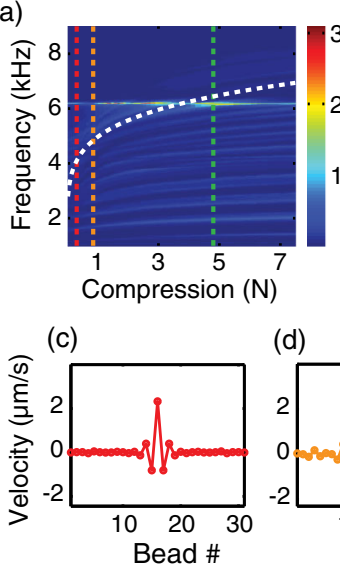

(d) (b)
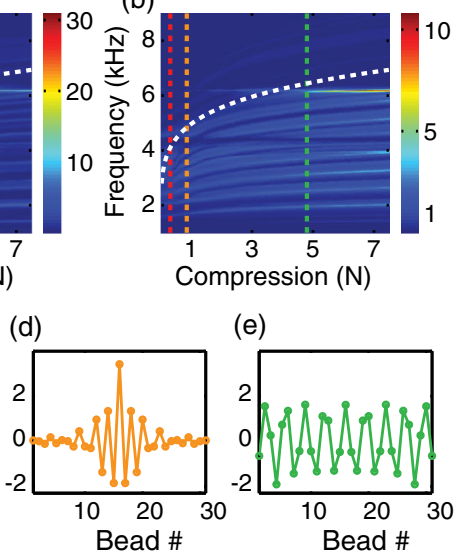

(e)

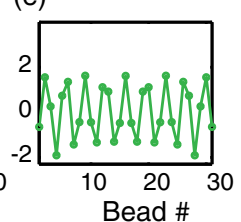

FIG. 2 (color online). The acoustic band and defect mode profile evolution. The color scale shows the steady-state amplitude at the embedded force sensors as a function of the frequency and the static compression, (a), next to the defect and, (b), at the crystal's edge. The defect mode, around $6.2 \mathrm{kHz}$, appears in the force sensor at the crystal's edge only after the band edge rises above the frequency of the defect mode (at $4 N$ ). The mode's frequency increases very slightly as the lattice is compressed. The dotted curve is the theoretical edge of the acoustic band. (c),(d), and (e) The local mode profile measured at three different compressions $(0.3 N, 0.9 N$, and $4.8 N)$, which are indicated as dotted vertical lines in (a) and (b). At higher compressions, shown in (e), the mode transitions to being completely extended and the dynamics are delocalized. The measurements are performed for half of the chain and we plot their mirrored image of the other half for clarity.

end of the chain. This sensor measures only the extended modes, i.e., effects far away from the defect. This sensor does not detect the defect mode until the band edge crosses the mode's frequency at approximately $4 N$. Beyond this compression, the defect mode becomes extended and the excitation propagates through the crystal. This shows that a single resonant defect can be tuned to arbitrary effective size.

More insight on the defect mode's dependence on compression can be seen in Figs. 2(c), 2(d), and 2(e), which show the mode's spatial profile at three increasing compressions, corresponding to the dashed vertical lines in Figs. 2(a) and 2(b). The left two panels [Figs. 2(c) and 2(d)] are measured at compressions while the mode is still localized, before the dynamics are extended. As the granular crystal is compressed and the acoustic band edge approaches the mode, the mode begins to delocalize, i.e., the exponential attenuation decreases. Above a certain compression the acoustic band rises above the defect mode frequency and the dynamics transition to being extended. An analogous tuning and transition has been achieved by geometrically altering defect cavity layers in phononic superlattices [17]. The rightmost panel [Fig. 2(e)] shows the extended mode profile at a higher compression. This reveals why there is

little force observed in Fig. 2(b) until high compressions. Supplemental video 2 in [33] presents an animation of this experimental result.

The underlying physics of the problem can be understood through an analytical model for a resonant defect between two semi-infinite generic lattices (see Supplemental Material [33]). We quantify the mode's spatial localization using an exponential decay factor $L$, defined as the ratio of the displacement amplitude between neighboring particles. This localization depends explicitly on the ratio $f_{c} / f_{d}$ of the band edge cutoff to the defect mode's frequencies,

$$
L=\frac{2 f_{d}^{2}}{f_{c}^{2}}\left[1 \pm \sqrt{1-\frac{f_{c}^{2}}{f_{d}^{2}}}\right]-1 .
$$

Therefore, a resonant defect mode's localization can be controlled by changing this ratio, which is accomplished through an external compression. However, since the defect mode is actually a mode of the entire lattice, the defect mode frequency also depends on the compression, or, equivalently, on the band edge $f_{c} \propto F_{0}^{1 / 6}$. The defect mode frequency can be found solving the implicit equation,

$$
\frac{m_{r}}{m_{0}}=\left(1-\frac{f_{d}^{2}}{f_{r}^{2}}\right)\left[\frac{m_{s}}{m_{0}}\left(1 \pm \sqrt{1-\frac{f_{c}^{2}}{f_{d}^{2}}}\right)-1\right] .
$$

Together these two equations describe the defect mode localization's dependence on compression, or, equivalently, on the acoustic band edge.

Figure 3(a) shows a comparison between the analytical solution in an infinite lattice with computational results for solving the eigenvalue problem for a finite chain of 31 particles. We can see the boundary effects play a negligible role until just before the mode theoretically delocalizes. Figure 3(a) shows three horizontal dashed lines, corresponding to the localization calculated for three different simple mass defect diameters, $11.1,14.3$, and $17.5 \mathrm{~mm}$. The smallest defect results in the greatest localization. This demonstrates that by changing the external compression, the resonant defect mode localization is similar to that of mass defects of differing sizes.

The experimental measurements of the localization factor as a function of different compressions are shown in Fig. 3(b), for over 26 independent tests. We fit these experimental results to our analytical model [Supplemental Material [33], Eq. (3)]. The analytical model accurately captures the localization's dependence on compression. We attribute the disagreement between the theoretical [Fig. 3(a)] and experimental [Fig. 3(b)] compression scale to friction effects, originating from the large mass of the resonator and significant contact area between the particles and supporting rods. 

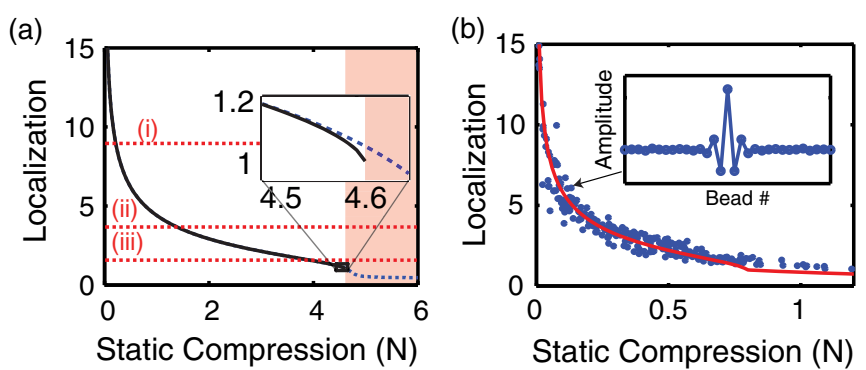

FIG. 3 (color online). Tunable localization of a resonant defect mode. (a) Localization factor predicted analytically (solid line) for an infinite lattice and calculated computationally for a 31 particle chain (dashed line). Three horizontal dashed lines (i), (ii), and (iii) show localizations for different defect diameters, 11.1, $14.3,17.5 \mathrm{~mm}$, respectively. In addition, all parameters used in the computation are those derived from the experimental setup. The inset in (a) is an enlarged view, showing the deviation of the computational and analytical results close to when the defect frequency and band edge meet. (b) The experimentally measured localization and a fit (red line) to the analytical prediction. Each experimental run is shifted along the compression axis to have the same zero point. The inset shows the mode profile used to measure the experimental localization for the run indicated by the arrow.

We computationally study resonant defect modes in a two-dimensional hexagonal lattice with nearest neighbor interactions. Although, the dynamics become more complex due to the presence of both transverse and longitudinal acoustic modes of the crystal, the defect modes' profiles can still be tuned through an external pressure. To understand the dynamics without a defect present, we first found the longitudinal and transverse branches applying periodic Bloch conditions (see Supplemental Material [33]) and solving the eigenvalue problem. Then we include the defect mode, and confirm that as the defect mode frequency passes below the upper edge of the longitudinal acoustic band, the mode delocalizes, similar to the case of the onedimensional granular crystal. Figure 4 illustrates the mode profile of a resonant defect in a finite lattice at two different
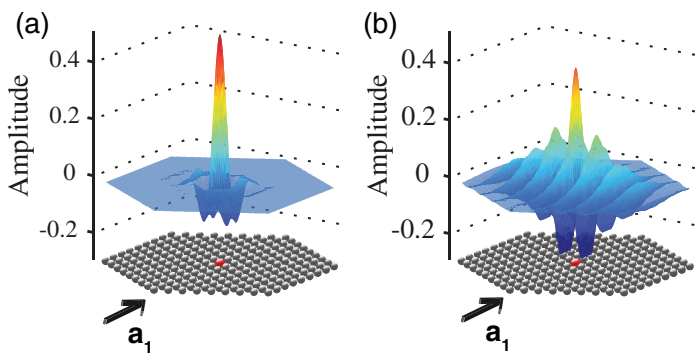

FIG. 4 (color online). Resonant defects in hexagonal lattices. The normalized mode profile due to a resonant defect (red) placed in a two dimensional hexagonal lattice (gray). We plot the amplitude of the particles' displacement in the nearest neighbor direction $a_{1}$. The mode becomes less localized as it goes from low (a), to high (b), isotropic compression. isotropic compressions (see Supplemental Material [33], video 1$)$.

Conclusion.-We have presented a method to actively control the localization of a defect mode in a nonlinear lattice of one and two dimensions. The results are easily extendable to three dimensions. The defect mode that results from a resonant defect has an additional degree of freedom that allows this tunability. This is the first time that a defect mode profile can be actively controlled and tuned without having to change the defect itself. In addition, since the same underlying principles of band tunability apply to a broad range of material systems, we anticipate that these results will impact current device and material applications.

We thank and acknowledge financial support from NSF CMMI CAREER (844540) and US-AFOSR (FA9550-121-0332).

* Corresponding author. daraio@ethz.ch

[1] N. I. Zheludev, Science 328, 582 (2010).

[2] L. Jae-Hwang, P. S. Jonathan, and L. T. Edwin, Adv. Mater. 24, 4782 (2012).

[3] H. J. Queisser and E. E. Haller, Science 281, 945 (1998).

[4] A. A. Balandin, Nat. Mater. 10, 569 (2011).

[5] Y. Wei, J. Wu, H. Yin, X. Shi, R. Yang, and M. Dresselhaus, Nat. Mater. 11, 759 (2012).

[6] A. Zandiatashbar, G.-H. Lee, S. Joo An, S. Lee, N. Mathew, M. Terrones, T. Hayashi, C. R. Picu, J. Hone, and N. Koratkar, Nat. Commun. 5, 3186 (2014).

[7] M. Wagner, Phys. Rev. 131, 1443 (1963).

[8] M. Torres, F. R. Montero de Espinosa, D. García-Pablos, and N. García, Phys. Rev. Lett. 82, 3054 (1999).

[9] J. D. Joannopoulos, S. G. Johnson, J. N. Winn, and R. D. Meade, Photonic Crystals: Molding the Flow of Light, 2nd ed. (Princeton University Press, Princeton, NJ, 2011).

[10] S. Rudykh and M. C. Boyce, Phys. Rev. Lett. 112, 034301 (2014).

[11] L. Brillouin, Wave Propagation in Periodic Structures: Electric Filters and Crystal Lattices (Dover, New York, 1953).

[12] Y. Man, N. Boechler, G. Theocharis, P. G. Kevrekidis, and C. Daraio, Phys. Rev. E 85, 037601 (2012).

[13] G. W. Milton and J. R. Willis, Proc. R. Soc. A 463, 855 (2007).

[14] M. Maldovan and E. L. Thomas, Appl. Phys. Lett. 88, 251907 (2006).

[15] S. Noda, A. Chutinan, and M. Imada, Nature (London) 407, 608 (2000).

[16] A. Yariv, Y. Xu, R. K. Lee, and A. Scherer, Opt. Lett. 24, 711 (1999).

[17] D. Schneider, F. Liaqat, E. H. El Boudouti, O. El Abouti, W. Tremel, H.-J. Butt, B. Djafari-Rouhani, and G. Fytas, Phys. Rev. Lett. 111, 164301 (2013).

[18] M. SoljaCiC and J. D. Joannopoulos, Nat. Mater. 3, 211 (2004). 
[19] E. B. Herbold, J. Kim, V. F. Nesterenko, S. Wang, and C. Daraio, Acta Mech. 205, 85 (2009).

[20] C. W. Wong, P. T. Rakich, S. G. Johnson, M. Qi, H. I. Smith, E. P. Ippen, L. C. Kimerling, Y. Jeon, G. Barbastathis, and S.-G. Kim, Appl. Phys. Lett. 84, 1242 (2004).

[21] M. Huang, H. Yan, C. Chen, D. Song, T. F. Heinz, and J. Hone, Proc. Natl. Acad. Sci. U.S.A. 106, 7304 (2009).

[22] M. Huang, H. Yan, T. F. Heinz, and J. Hone, Nano Lett. 10, 4074 (2010).

[23] W. Yan, W.-Y. He, Z.-D. Chu, M. Liu, L. Meng, R.-F. Dou, Y. Zhang, Z. Liu, J.-C. Nie, and L. He, Nat. Commun. 4, 2159 (2013).

[24] G. Fedorov, P. Barbara, D. Smirnov, D. Jiménez, and S. Roche, Appl. Phys. Lett. 96, 132101 (2010).

[25] J. Hwang, M. H. Song, B. Park, S. Nishimura, T. Toyooka, J. W. Wu, Y. Takanishi, K. Ishikawa, and H. Takezoe, Nat. Mater. 4, 383 (2005).

[26] S. Y. T. Tzeng, C. N. Chen, and Y. Tzeng, Liq. Cryst. 37, 1221 (2010).
[27] N. Boechler, J. Yang, G. Theocharis, P. G. Kevrekidis, and C. Daraio, J. Appl. Phys. 109, 074906 (2011).

[28] H. Hertz, in Journal für die reine und angewandte Mathematik (Crelle's Journal, 1882), p. 156.

[29] N. Boechler, G. Theocharis, and C. Daraio, Nat. Mater. 10, 665 (2011).

[30] N. Boechler, G. Theocharis, S. Job, P. G. Kevrekidis, M. A. Porter, and C. Daraio, Phys. Rev. Lett. 104, 244302 (2010).

[31] A. Spadoni and C. Daraio, Proc. Natl. Acad. Sci. U.S.A. 107, 7230 (2010).

[32] G. Gantzounis, M. Serra-Garcia, K. Homma, J. M. Mendoza, and C. Daraio, J. Appl. Phys. 114, 093514 (2013).

[33] See Supplemental Material at http://link.aps.org/ supplemental/10.1103/PhysRevLett.113.185503, which includes Refs. [35,36].

[34] ASM International, Metals Handbook 10th ed. (ASM INternational, Materials Park, OH, 1990).

[35] C. T. Walker and R. O. Pohl, Phys. Rev. 131, 1433 (1963).

[36] G. W. Milton and J. R. Willis, Proc. R. Soc. A 463, 855 (2007). 